\title{
How a Kinase Inhibitor Withstands Gatekeeper Residue Mutations
}

\section{Supplementary Information}

\author{
Jagannath Mondal, ${ }^{* \dagger}$ Pratyush Tiwary, ${ }^{\ddagger}$ and B. J. Berne ${ }^{*, \ddagger}$ \\ $\dagger$ Tata Institute of Fundamental Research, Center for Interdisciplinary sciences, Hyderabad, \\ 500075, India \\ $\ddagger$ Department of Chemistry, Columbia University, New York 10027, USA. \\ E-mail: jmondal@tifrh.res.in; bb8@columbia.edu
}




\section{Justification for using IC50}

The relation between IC50 and ligand binding affinity, $K_{i}$, is given by the Cheng-Prusoff equation $^{1,2}$ :

$$
K_{i}=\frac{I C 50}{\left(1+[S] / K_{m}\right]},
$$

where $[S]=$ Substrate concentration, and $K_{m}=$ Michelis Menten constant. When $[S]<<K_{m}$, $K_{i} \simeq I C 50$. Interestingly, experimental values ${ }^{3}$ of $K_{m}$ for ATP binding to either wildtype or T338M-mutated kinase $(15$ and $1 \mu \mathrm{M})$ (the ligands studied in our paper) are orders of magnitude larger than the substrate concentration $[S] \approx 0.1 \mu \mathrm{M})$. Hence, for all practical purposes, we can approximate $K_{i} \simeq I C 50$ so that

$$
\Delta \Delta G=R T \ln \left[\frac{\left.K_{i} \text { (wildtype }\right)}{K_{i}(\text { mutant })}\right] \simeq R T \ln \left[\frac{(I C 50)_{\text {wildtype }}}{(I C 50)_{\text {mutant }}}\right]
$$

This gives the rationale for using the ratio of $I C 50$ values to obtain an experimental estimate of $\Delta \Delta \mathrm{G}$, in the absence of experimental $K_{i}$ data for certain cases from the experiments of Getlik et al. ${ }^{3}$

Eq.(2) has been extensively used to determine $\Delta \Delta G$. For example in a highly cited paper, Jorgensen and coworkers ${ }^{4}$ have used $I C 50$ identical to estimate the experimental relative binding free energies of several small molecules to COX-1 and COX-2 and compared this with their computed relative binding free energy, as we do for our system here. The excellent agreement between computed and experimental relative binding free energy (estimated from ratio of IC50) gives evidence of usefulness of this approach. On a similar note, in a recent paper $^{5}$ Friesner and coworkers at Schrodinger Inc have made extensive use of the above IC50-based equation to estimate the experimental relative binding free energies from IC50 for a large set of 200 ligands. The impressive agreement between their experimental and in silico relative binding free energies cannot be accidental.Other papers ${ }^{6,7}$ utilize this approach as well. Eq,(2) is obviously a useful approximation when the mechanism of protein-ligand binding is similar for the different ligands. This is the case for our current protein-ligand 
system.

\section{References}

(1) Yung-Chi, C.; Prusoff, W. H. Biochem. Pharmacol. 1973, 22, 3099 - 3108.

(2) Cer, R. Z.; Mudunuri, U.; Stephens, R.; Lebeda, F. J. Nuc. Acids Res. 2009, 37, W441W445.

(3) Getlik, M.; Grütter, C.; Simard, J. R.; Klüter, S.; Rabiller, M.; Rode, H. B.; Robubi, A.; Rauh, D. J. Med. Chem. 2009, 52, 3915-26.

(4) Price, M. L. P.; ; Jorgensen, W. L. J. Am. Chem. Soc. 2000, 122, 9455-9466.

(5) Wang, L. et al. J. Am. Chem. Soc. 2015, 137, 2695-2703.

(6) Luccarelli, J.; Michel, J.; Tirado-Rives, J.; Jorgensen, W. L. J. Chem. Theory Comput. 2010, 6, 3850-3856.

(7) Michel, J.; Verdonk, M. L.; Essex, J. W. J. Med. Chem. 2006, 49, 7427-7439. 


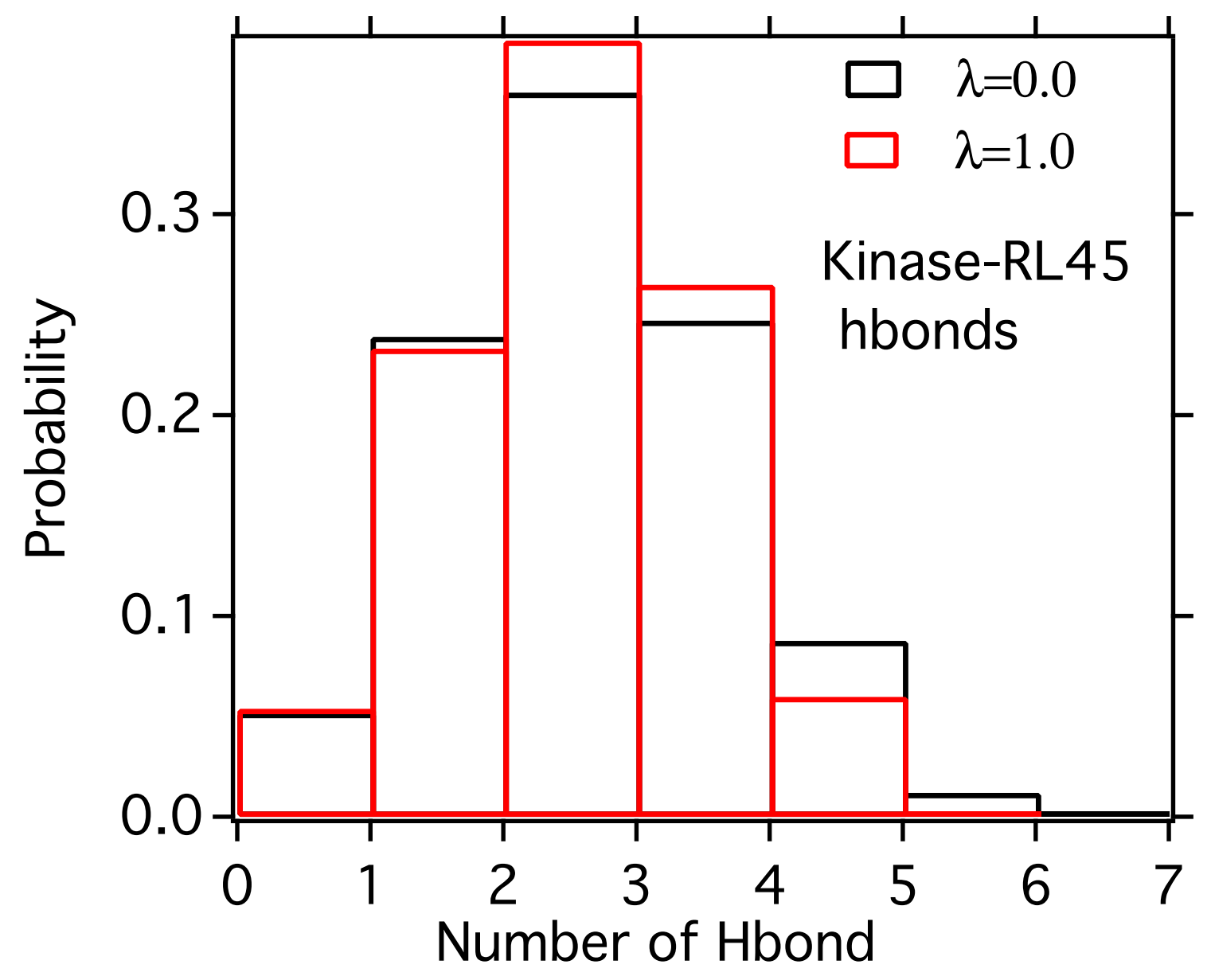

Figure S1: Comparison of distribution of hydrogen bonds between RL45 and kinase in wild type (THR338, $\lambda=0$ ) and mutant (MET338, $\lambda=1.0$ ) 


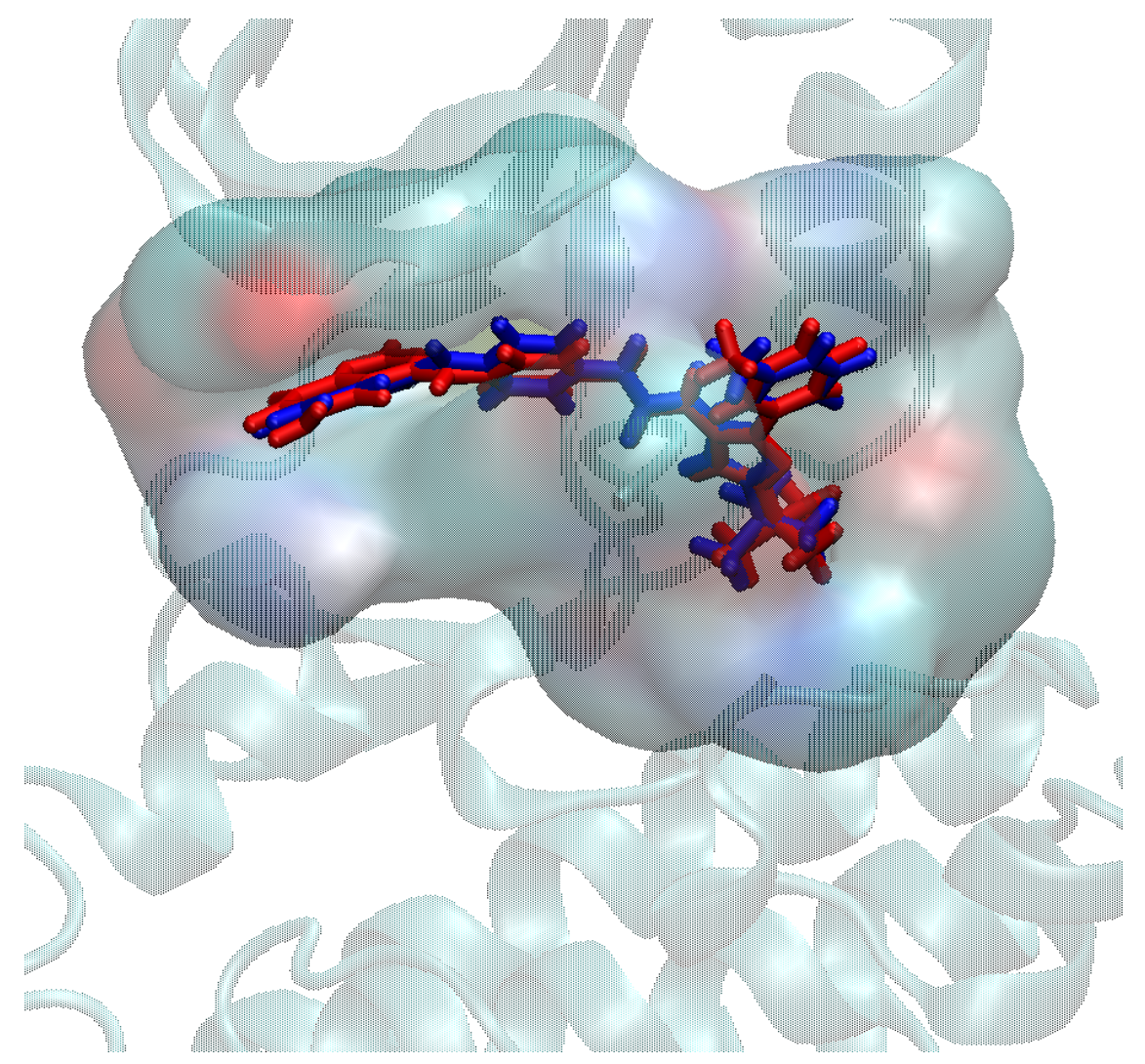

Figure S2: Overlay of simulated and crystal data of RL45 bound to mutated kinase. The RMSD is less than $1 \mathrm{~nm}$. 


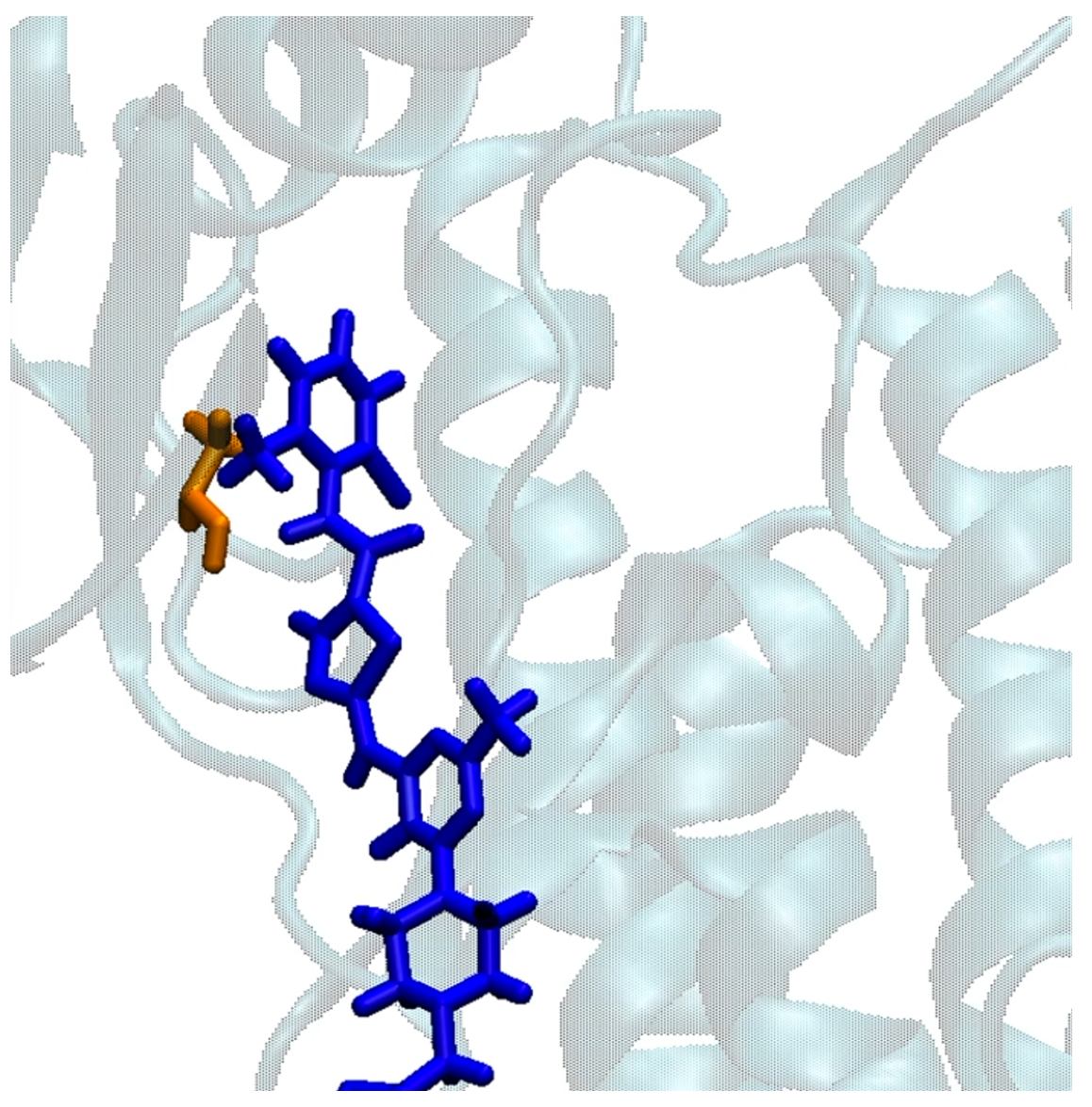

Figure S3: Thumbnail for 'Movie 1' showing the inflexibility of phenyl ring of dasatinib to avoid steric overlap upon mutation. Please look into the uploaded video file for the trajectory. 


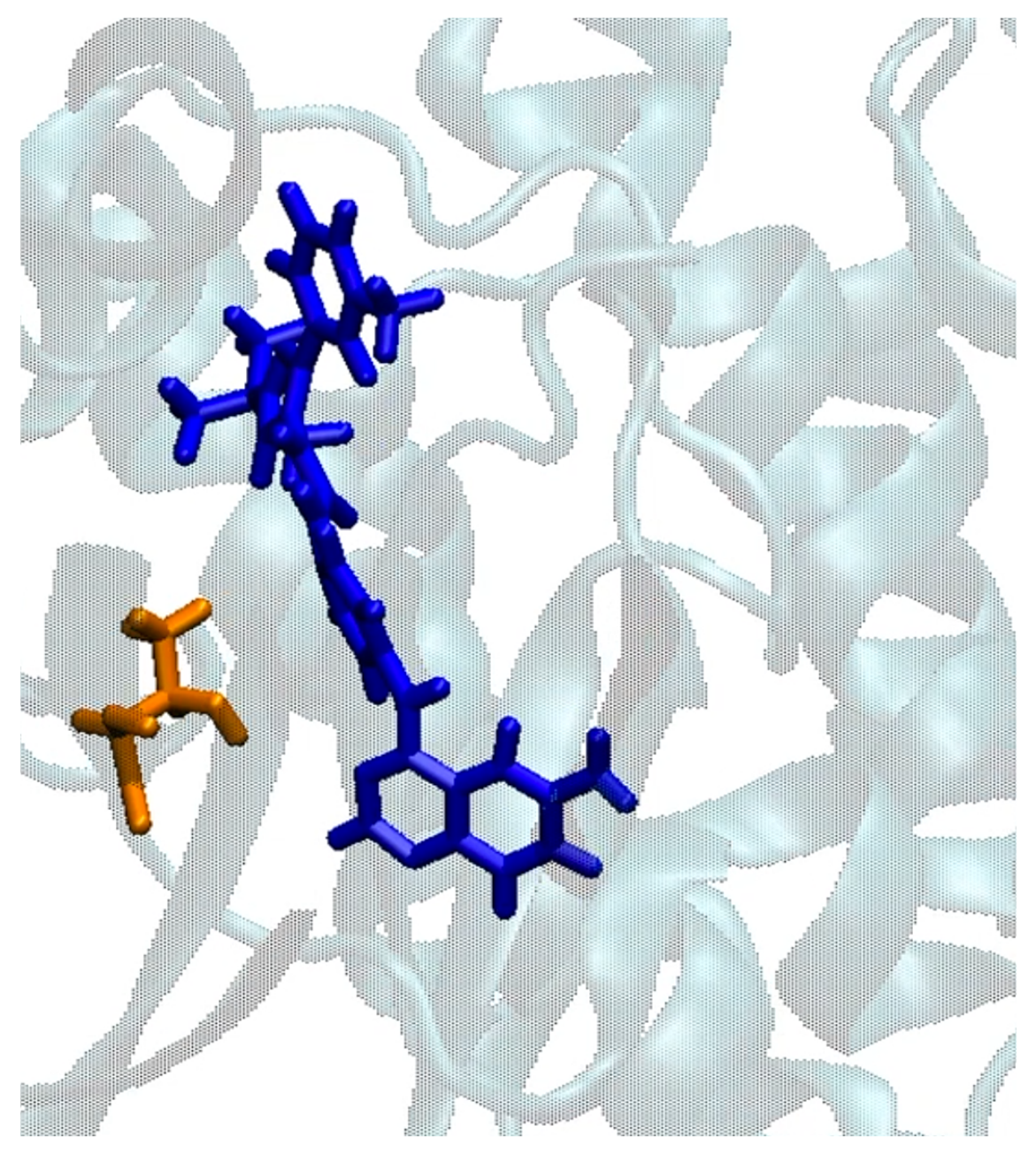

Figure S4: Thumbnail for 'Movie 2' showing the remarkable flexibility of central phenyl ring of RL45 in adapting to mutation. Please look into the uploaded video file for the trajectory. 


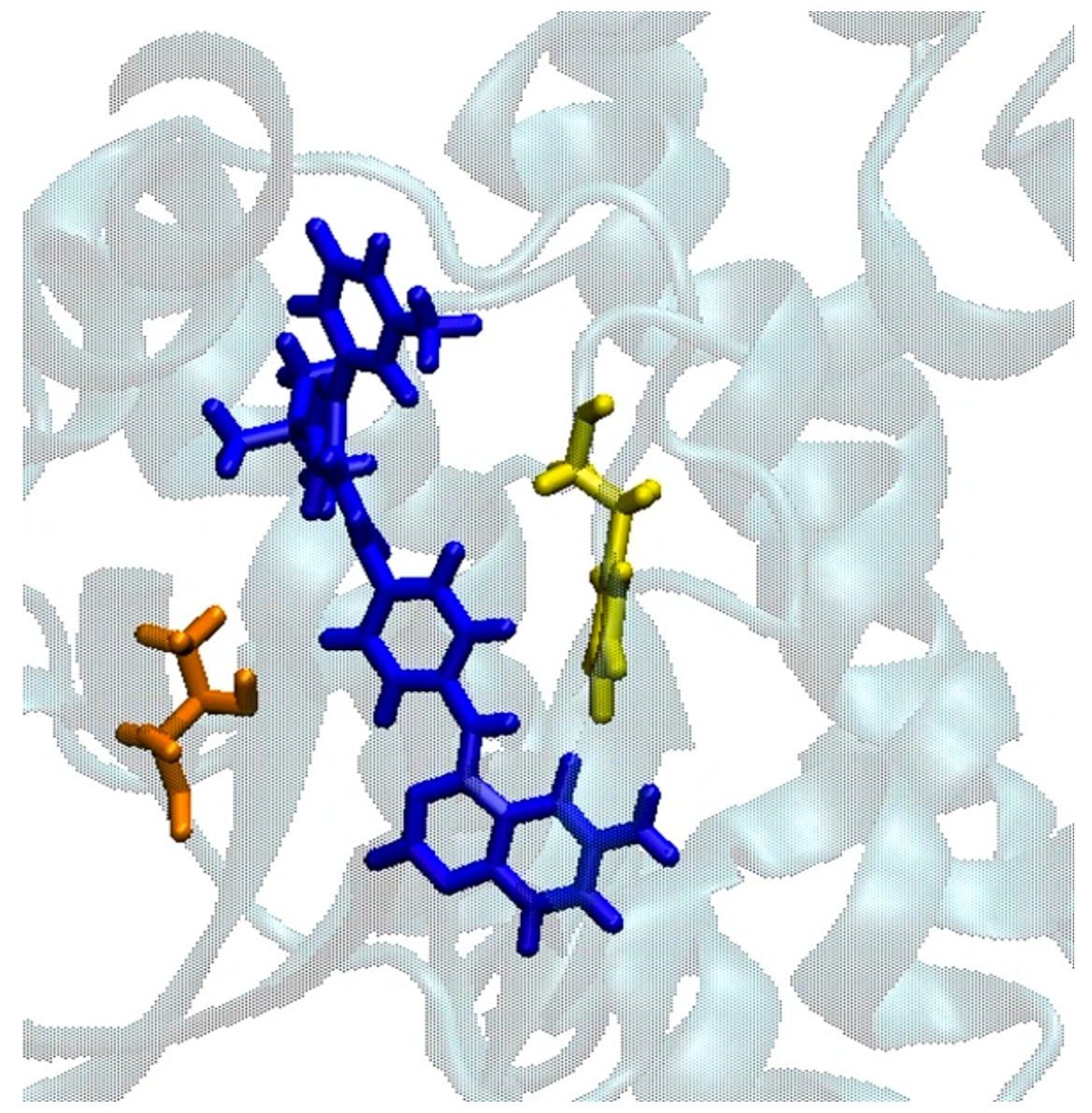

Figure S5: Thumbnail for 'Movie 3' showing the persistence of edge-to-face orientation of central phenyl ring of RL45 with Phe405. Please look into the submitted video file for the trajectory. 